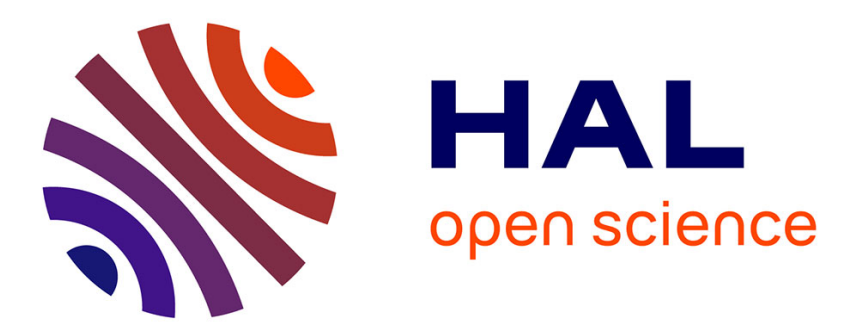

\title{
Optical properties of GaN nanowires grown on chemical vapor deposited-graphene
}

\author{
L. Mancini, M Morassi, C. Sinito, O Brandt, L. Geelhaar, Hyun-Gyu Song, \\ Yong-Hoon Cho, N Guan, Antonella Cavanna, Joanna Njeim, et al.
}

\section{- To cite this version:}

L. Mancini, M Morassi, C. Sinito, O Brandt, L. Geelhaar, et al.. Optical properties of GaN nanowires grown on chemical vapor deposited-graphene. Nanotechnology, 2019, 30 (21), pp.214005. 10.1088/1361-6528/ab0570 . hal-02349609

\section{HAL Id: hal-02349609 \\ https://hal.science/hal-02349609}

Submitted on 5 Nov 2019

HAL is a multi-disciplinary open access archive for the deposit and dissemination of scientific research documents, whether they are published or not. The documents may come from teaching and research institutions in France or abroad, or from public or private research centers.
L'archive ouverte pluridisciplinaire HAL, est destinée au dépôt et à la diffusion de documents scientifiques de niveau recherche, publiés ou non, émanant des établissements d'enseignement et de recherche français ou étrangers, des laboratoires publics ou privés. 


\section{Optical properties of GaN Nanowires grown on}

\section{chemical vapor deposited-Graphene}

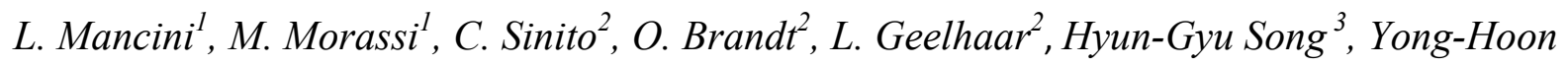

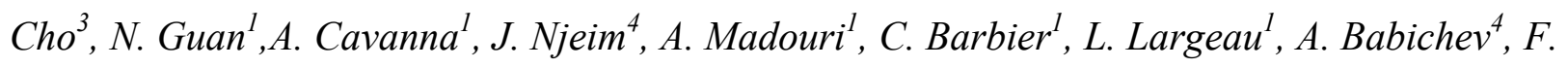

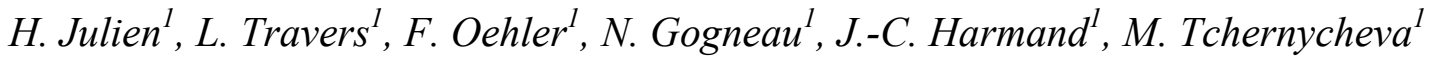

${ }^{1}$ Centre de Nanosciences et de Nanotechnologies (C2N) sites Orsay and Marcoussis, UMR9001 CNRS, University Paris Sud, University Paris Saclay, France

${ }^{2}$ Paul-Drude-Institut für Festkörperelektronik, Hausvogteiplatz 5-7,Leibniz-Institut im

Forschungsverbund Berlin e. V., 10117 Berlin, Germany

${ }^{4}$ GeePs Group of Electrical Engineering-Paris, UMR CNRS 8507, Sorbonne Université, Centrale Supelec, Univ. Paris-Sud, Université Paris Sacalay, 11 rue Joliot Curie, 91192 Gif-Sur-Yvette, France.

${ }^{5}$ ITMO University, 197101 St. Petersburg, Russia

*Corresponding author: maria.tchernycheva@u-psud.fr phone: +33 (0) 169154051 


\section{ABSTRACT:}

Optical properties of GaN nanowires grown on chemical vapor deposited graphene transferred on an amorphous support are reported. The growth temperature was optimized to achieve a high nanowire density with a perfect selectivity with respect to a $\mathrm{SiO}_{2}$ surface. The growth temperature window was found to be rather narrow $(815 \pm 5){ }^{\circ} \mathrm{C}$. Steady-state and time-resolved photoluminescence from $\mathrm{GaN}$ nanowires grown on graphene was compared with the results for GaN nanowires grown on conventional substrates within the same molecular beam epitaxy (MBE)reactor showing a comparable optical quality for different substrates. Growth at temperatures above $820^{\circ} \mathrm{C}$ led to a strong NW density reduction accompanied with a diameter narrowing. This morphology change leads to a spectral blueshift of the donor bound exciton emission line due to either surface stress or dielectric confinement. Graphene multi-layered microdomains were explored as a way to arrange $\mathrm{GaN}$ nanowires in a hollow hexagonal pattern. The nanowires grown on these domains show a luminescence spectral linewidth as low as $0.28 \mathrm{meV}$ (close to the set-up resolution limit).

KEYWORDS: GaN growth on graphene, van der Waals epitaxy, nanowires, graphene microdomains, photoluminescence

\section{INTRODUCTION :}

Nitrides are key materials for applications such as solid state lighting or high power electronics. Because of the absence of cost-efficient native substrates, nitride layers are usually synthesized by heteroepitaxy on sapphire, $\mathrm{SiC}$ or $\mathrm{Si}$. The main issue of this approach is the generation of threading dislocations due to the lattice mismatch with the crystalline substrates. To circumvent this problem, one of the promising approaches, which has recently emerged in the community, is the use of the so-called van der Waals (vdW) epitaxy on $2 \mathrm{D}$ materials ${ }^{1}$. The term "vdW epitaxy" initially refers to the growth of materials without covalent bonds out of the growth plane, which means that the consecutive layers are bonded only by vdW forces. Recently it has been claimed that the growth of crystals like GaN or GaAs (with $\mathrm{sp}^{3}$ orbital hybridisation, which normally have out of plane bonds) on graphene (with $\mathrm{sp}^{2}$ orbital hybridization and no out of plane bonds) can follow the vdW

epitaxy mechanism ${ }^{2}$. The interest of the vdW epitaxy for device applications is that the absence of covalent bonds with the substrate potentially releases the requirement for lattice matching. Therefore, a strong reduction of the dislocation density can be expected for this growth mode. The additional advantage of the $\mathrm{vdW}$ bonding is that the mechanical strength of the interface between the layer and the substrate is weak, so that the grown semiconductor layer can be mechanically peeled-off and transferred to a different host substrate such as metal for a better thermal management ${ }^{3}$ or plastic for mechanically flexible devices ${ }^{4}$.

Following these promising predictions, the growth of $\mathrm{GaN}$ thin films on layered materials such as graphene $\mathrm{e}^{5}$ or h-BN ${ }^{6-9}$ is today actively explored. In particular, GaN film growth and mechanical 
peel-off was demonstrated leading to spectacular demonstrations of LEDs ${ }^{5,6}$, HEMTs by mechanical transfer ${ }^{7,9}$ or metal-semiconductor-metal photodiodes ${ }^{10}$. However, the expected reduction of defect density in thin films was not achieved: a dislocation density around $10^{9}-10^{10}$ $\mathrm{cm}^{-2}$ was observed ${ }^{5-7,9,10}$. The interest has then turned to the growth of nanowires (NWs) on 2D materials since these nano-objects are known to be free from threading dislocations even on highly lattice-mismatched substrates. Metal-organic vapor phase epitaxy was applied by different groups to elaborate catalyst-free NWs on graphene ${ }^{11-14}$. Later, self-assembled GaN NWs by plasmaassisted molecular beam epitaxy (PA-MBE) were reported on CVD-graphene transferred to an amorphous material ${ }^{15,16}$ or to a crystalline substrate ${ }^{17}$, on graphite ${ }^{18}$ and on epitaxial multi-layered graphene on $\mathrm{SiC}^{19}$. In particular, it has been shown that when $\mathrm{GaN}$ is grown on a CVD-graphene layer transferred to a $\mathrm{SiO}_{2} / \mathrm{Si}$ substrate, an epitaxial order is observed with respect to the graphene lattice and growth selectivity with respect to the $\mathrm{SiO}_{2}$ surface can be obtained ${ }^{15}$. Contrary to the epitaxial multi-layered graphene on $\mathrm{SiC}, \mathrm{CVD}$-graphene grown on a copper foil is a cheap material which can be produced on a large scale, making it attractive for device applications.

Luminescence properties of the GaN material grown on CVD-graphene are of tremendous importance in view of the potential optoelectronic applications. However, no systematic optical studies of NWs grown on 2D materials have been reported so far. The only reported luminescence of GaN NWs on CVD-graphene was rather broad (14 meV reported in ${ }^{15}$ to be compared with 2.3 $\mathrm{meV}$ for GaN NWs on the epitaxial multi-layered graphene ${ }^{19}$ ). The objective of the present work is to benchmark the optical properties of the GaN NWs grown on CVD-graphene against the ones of NWs grown on conventional substrates.

Here we analyse the optical properties of GaN NWs grown by PA-MBE on different CVDgraphene patches transferred to an amorphous $\mathrm{SiO}_{2}$ support. The steady-state and time-resolved luminescence is compared with the one for GaN NWs grown on other crystalline ( $\mathrm{SiN} / \mathrm{Si}(111)$ and AlN/Si(111)) and amorphous $\left(\mathrm{SiO}_{2} / \mathrm{Si}(100)\right)$ substrates. All growths were performed subsequently and within the same MBE reactor to avoid any influence of the chamber background. The results show that the optical properties of GaN NW arrays grown on graphene are comparable to that of wires grown on the other types of substrates, both in terms of energy of emission and of temporal dynamics. Large area graphene patches and sub- $\mu \mathrm{m}$ graphene domains are investigated as growth substrates. Growth selectivity, NW morphology and optical characteristics are explored as a function of growth temperature. For graphene micro-domains containing a reduced number of NWs, a narrow linewidth of $0.28 \mathrm{meV}$, close to the set-up resolution limit, is achieved.

\section{GaN nanowire growth and growth selectivity}

GaN NWs were grown by PA-MBE on AlN/Si(111), on nitridated $\mathrm{Si}$ (in the following referred to as $\mathrm{SiN} / \mathrm{Si}(111))$, on $\mathrm{SiO}_{2} / \mathrm{Si}(100)$ and on graphene $/ \mathrm{SiO}_{2}(300 \mathrm{~nm}) / \mathrm{Si}(100)$ substrates. The graphene transfer conditions are described in the Methods section. The NW growth was performed in a RIBER Compact 12 MBE system equipped with a radio-frequency (RF) plasma $\mathrm{N}$ source. The substrate temperature was monitored using an optical pyrometer, calibrated before each growth by 
following the disappearance of the $\mathrm{Si}(111)-(7 \times 7)$ reconstruction at $830{ }^{\circ} \mathrm{C}^{20}$ using reflection highenergy electron diffraction (RHEED). For all growths, the plasma cell was operated at $0.57 \mathrm{sccm}$ $\mathrm{N}_{2}$ flow and $400 \mathrm{~W}$ RF power conditions (corresponding to a GaN equivalent planar growth rate of 0.70 monolayer/s (ML/s)). The total growth time was set to 3 hours. To obtain comparable NW morphologies on different substrates, the growth temperature and the V/III ratio were adjusted. Fig.1 (a), (b), (c) and (d) shows $45^{\circ}$ tilted scanning electron microscopy (SEM) images of the NWs grown on the different substrates under the following growth conditions:

a. AlN/Si(111) substrate: the growth was initiated by depositing a thin (2-3 nm) AlN buffer layer at $620^{\circ} \mathrm{C}$ on an oxide-free $\mathrm{Si}(111)$ substrate. This was done following a previously reported procedure [Largeau 2012]. The GaN NWs were grown at $790{ }^{\circ} \mathrm{C}$ with a V/III ratio of 1.36. The resulting NW diameter is $50 \pm 10 \mathrm{~nm}$ and the length is $1 \pm 0.1 \mu \mathrm{m}$

b. $\mathrm{SiN} / \mathrm{Si}(111)$ substrate: before $\mathrm{NW}$ growth, the oxide-free $\mathrm{Si}(111)$ surface was exposed to $\mathrm{N}$ plasma for 10 minutes in order to form a compact 2-3 $\mathrm{nm} \mathrm{SiN}$ layer. Then the temperature was adjusted to $810^{\circ} \mathrm{C}$ and the NWs were grown with V/III ratio of 1.8. The resulting NW diameter is $60 \pm 10 \mathrm{~nm}$ and the length is $1.55 \pm 0.2 \mu \mathrm{m}$.

c. $\mathrm{SiO}_{2}(300 \mathrm{~nm}) / \mathrm{Si}(100): 300 \mathrm{~nm}$ thick $\mathrm{SiO}_{2}$ layer was obtained by dry oxidation. The NW growth temperature was set to $800{ }^{\circ} \mathrm{C}$ and the $\mathrm{V} / \mathrm{III}$ ratio was 1.36 . The resulting $\mathrm{NW}$ diameter is $68 \pm 10 \mathrm{~nm}$ and the length is smaller than for the other samples $0.66 \pm 0.2 \mu \mathrm{m}$.

d. Graphene/ $\mathrm{SiO}_{2}(300 \mathrm{~nm}) / \mathrm{Si}(100)$ substrates (both macroscopic graphene patches and monocrystalline micro-domains): best growth conditions for this kind of systems were assessed by varying the growth temperature within the $805-820^{\circ} \mathrm{C}$ temperature range. The $\mathrm{V} / \mathrm{III}$ ratio was fixed to 1.1 for all the samples. Optimal selectivity and a high NW density (Fig. 1 (d)) were obtained at $815^{\circ} \mathrm{C}$. The resulting NW diameter at this temperature is $60 \pm$ $25 \mathrm{~nm}$ and the length is $1.1 \pm 0.1 \mu \mathrm{m}$.

The use of a non-crystalline $\mathrm{SiO}_{2}$ substrate resulted in a lack of in-plane orientation of the $\mathrm{NWs}{ }^{15}$, evidenced by rings in the RHEED pattern, and in a lower NW verticality, as can be seen in figure 1(c). By contrast, $\mathrm{SiN} / \mathrm{Si}, \mathrm{AlN} / \mathrm{Si}$ and graphene $/ \mathrm{SiO}_{2} / \mathrm{Si}$ substrates resulted in the growth of dense vertical arrays with a well-defined in-plane orientation of the NWs.

A series of four samples was grown on $1 \mathrm{~cm}^{2}$ graphene patches by fixing the growth temperature at $805{ }^{\circ} \mathrm{C}, 810{ }^{\circ} \mathrm{C}, 815{ }^{\circ} \mathrm{C}$ and $820^{\circ} \mathrm{C}(3 \mathrm{~h}$ total deposition time, V/III ratio 1.1) as illustrated in Fig. 2. At the lowest substrate temperature $\left(805^{\circ} \mathrm{C}\right.$, figure 2 (a)), well-developed GaN NWs are grown in high density on both the $\mathrm{SiO}_{2}$ surface and on graphene. Importantly, the NWs on graphene are highly vertical and show a well-defined in-plane orientation, while on $\mathrm{SiO}_{2}$, they are slightly tilted with a random in-plane orientation. The growth is quasi-selective at $810^{\circ} \mathrm{C}$, with few short NWs nucleating on $\mathrm{SiO}_{2}$ and well-developed NWs on the graphene (Fig. 2 (b)). A perfect selectivity is attained at $815^{\circ} \mathrm{C}$, with no NWs on the $\mathrm{SiO}_{2}$ areas and high-density vertical NWs on the area supposedly covered by graphene (Fig. 2 (c)). However, when the temperature is increased by only 5 degrees, the selectivity is maintained, but the NW density decreases sharply $\left(820^{\circ} \mathrm{C}\right.$, Fig. 2 (d)). Following the evolution of the NW morphology from the center to the edge of this sample, 
where the effective temperature is higher, the NW density dropped below $0.1 \mathrm{NW} / \mu \mathrm{m}^{2}$ (Fig. 2 (e)) and then the growth was completely inhibited.

From these results, it is clear that the GaN NWs grow preferentially on graphene in the $810-820{ }^{\circ} \mathrm{C}$ temperature range. However, for nucleation at these temperatures, the first specular RHEED spot appears systematically after a deposition time of about 1 hour at $810 \mathrm{C}^{\circ}$ and 1 hour 20 minutes at $815^{\circ} \mathrm{C}$. This long incubation time indicates a low nucleation probability (per area and time units) on graphene, most probably associated to a very high desorption rate of Ga from the graphene surface. This statement is supported by the fact that at lower growth temperature, Ga desorption decreases, and NW nucleation accelerates (note that this holds for any substrate, e.g. for $\mathrm{Si}^{19}$ ). Since the NW incubation time is long and it increases exponentially with temperature ${ }^{19,21}$, a small increase of temperature is sufficient to inhibit the growth also on graphene.

\section{Comparison of the luminescence properties between nanowires grown on different substrates}

Numerous photoluminescence (PL) studies addressed the optical properties of GaN NWs grown by PA-MBE on conventional substrates. Robins et al. ${ }^{22}$ reported donor-bound excitonic transition $\left(\mathrm{D}^{0}, \mathrm{X}_{\mathrm{A}}\right)$ with a width of around $3 \mathrm{meV}$, which they ascribed to strain inhomogeneity. Corfdir et al. ${ }^{23}$ reported comparable linewidth of around $5 \mathrm{meV}$ for both donor bound exciton peaks $(3.47 \mathrm{eV})$ and peaks around $3.45 \mathrm{eV}$, and ascribed this width to the energy dispersion of bound-exciton states as a function of their distance from the surface. Both Calleja et al. ${ }^{24}$ and van Nostrand et al. ${ }^{25}$ reported linewidths for GaN NWs exceeding $1 \mathrm{meV}$. Brandt et al. ${ }^{26}$ measured a near-band edge (NBE) emission linewidth as low as $0.3 \mathrm{meV}$ (limit of the measurement setup's spectral resolution) for single dispersed NWs. They interpreted the $2-3 \mathrm{meV}$ linewidth of the NW ensemblein the same way as Corfdir et al. ${ }^{23}$, i.e., as being caused by the energy difference between excitons bound to bulk and surface donors. Similar values for NW ensembles were reported by Pfüller et al. ${ }^{27}$ ( 2 $\mathrm{meV})$ and Jenichen et al. ${ }^{28}(1.6 \mathrm{meV})$. Corfdir et al. ${ }^{29}$ observed a narrowing of the linewidth from 1.2 to $0.4 \mathrm{meV}$ with increasing donor density, which was attributed to the ionization of surface donors and the resulting absence of surface donor bound excitons. For single dispersed NWs from these ensembles, Corfdiret al. ${ }^{30}$ observed transitions from individual donor-bound excitons with a (resolution limited) linewidth of $0.25 \mathrm{meV}$, and transition energies scattering over a few meV. Free exciton transitions were observed to be almost one order of magnitude broader. Finally, Zettler et al. ${ }^{31}$ obtained a NBE emission linewidth as low as $0.7 \mathrm{meV}$ on NW ensembles grown at higher temperature with a two-step method.

Fig. 1 displays the steady-state micro-photoluminescence ( $\mu \mathrm{PL})$ spectra acquired from NW ensembles grown on reference substrates, namely (e) $\mathrm{AlN} / \mathrm{Si}(111)$, (f) $\mathrm{SiN} / \mathrm{Si}(111)$, (g) bare $\mathrm{SiO}_{2} / \mathrm{Si}(100)$, and (h) from a NW array grown at $815^{\circ} \mathrm{C}$ on a $1 \mathrm{~cm}^{2}$ graphene patch transferred to $\mathrm{SiO}_{2} / \mathrm{Si}(100)$ template. All spectra were collected within the same experimental conditions described in the methods section. The samples show a NBE emission dominated by the donorbound exciton recombination process $\left(\mathrm{D}^{0}, \mathrm{X}_{\mathrm{A}}\right)$, peaked around $3.471 \mathrm{eV}$ with a few meV spectral broadening (full width at half maximum (FWHM) goes from $1.1 \mathrm{meV}$ for the AlN/Si(111) substrate 
to $2.6 \mathrm{meV}$ for $\mathrm{NWs}$ on graphene/ $\mathrm{SiO}_{2} / \mathrm{Si}(100)$ ). Contributions from the free exciton recombination, free exciton $\mathrm{A}$ transition $\left(\mathrm{F}, \mathrm{X}_{\mathrm{A}}\right)$ at $3.478 \mathrm{eV}$ (for all systems) and donor bound exciton recombination $3.475 \mathrm{eV}\left(\mathrm{D}^{0}, \mathrm{X}_{\mathrm{B}}\right)$ (mainly for the NWs on graphene) are also observed. The spectral signatures of grown-on-graphene NWs were further addressed by performing $\mu \mathrm{PL}$ analysis on single dispersed NWs (Fig. 1 (i)). Fig. 1 (j) reports a spectrum acquired from an individual NW. The emission is characterized by the dominating $\left(\mathrm{D}^{0}, \mathrm{X}_{\mathrm{A}}\right)$ line with a spectral linewidth (FWHM) of $1.3 \mathrm{meV}$ and by additional contributions from $\left(\mathrm{F}, \mathrm{X}_{\mathrm{A}}\right)$ and $\left(\mathrm{D}^{0}, \mathrm{X}_{\mathrm{B}}\right)$. It should be noted that 1.3 $\mathrm{meV}$ is a relatively large spectral broadening for a single emitting NW if compared to line widths reported in literature for analogous systems (e.g. ${ }^{30}$ ) and to the broadening observed in this work for few standing NWs (see discussion of optical properties of GaN NWs grown on graphene microdomains). The larger broadening was tentatively ascribed to the interaction between the dispersed NWs and the $\mathrm{SiO}_{2}$ substrate.

In comparison with the previous report by Kumaresan et al. ${ }^{15}$, a much smaller broadening of the $\left(\mathrm{D}^{0}, \mathrm{X}_{\mathrm{A}}\right)$ is observed for the NWs ensemble (2.6 meV (Fig. 1 (i)) compared to $14 \mathrm{meV}$, in better agreement with what reported by Fernández-Garrido and collaborators ${ }^{32}$ ). In this previous work, it was stated that the important broadening was due to a large residual doping coming from the MBE growth chamber but not from contaminants induced by the graphene transfer. The present analyses confirm that narrow NBE linewidth can be obtained for GaN NWs grown on CVDgraphene.

A broader emission peaked at $3.45 \mathrm{eV}$ characterizes the case of the $\mathrm{AlN} / \mathrm{Si}(111)$ substrate and is absent in the other systems. Such emission is a well-known feature of GaN NWs and it has been recently ascribed to the presence of inversion domain boundaries (IDB*) ${ }^{33,34}$. In $2 \mathrm{D}$ GaN layers this type of defects is usually assigned to the growth on monolayer-stepped $\operatorname{Si}(111)$ surfaces ${ }^{35}$. A similar mechanism could, in principle, apply also to GaN NWs grown on AlN/Si(111) substrates, but further investigations are needed to confirm this point. We note that the IDB signature is absent not only in PL spectra acquired at different positions of the graphene patch shown in Fig. 1 (d), but also for GaN NWs grown on graphene patches at different growth temperatures and for NWs grown on graphene micro-domains. We conclude that the IDB defects do not appear in GaN NWs grown on graphene at any of the explored conditions (consistently with what concluded in ${ }^{32}$ for $\mathrm{GaN}$ NWs on multilayer graphene). 
(a)

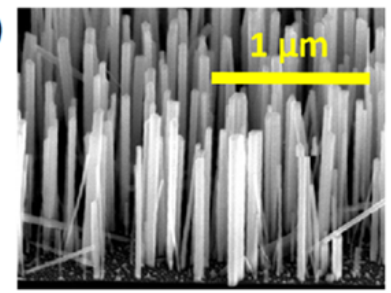

(b)

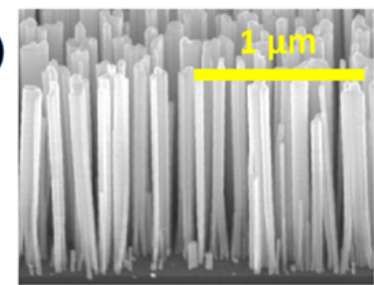

(c)

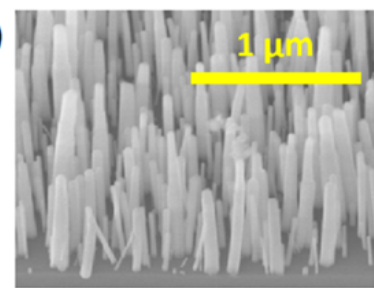

(d)

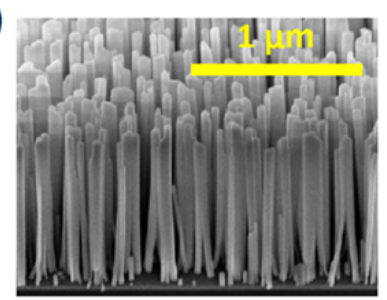

(i)

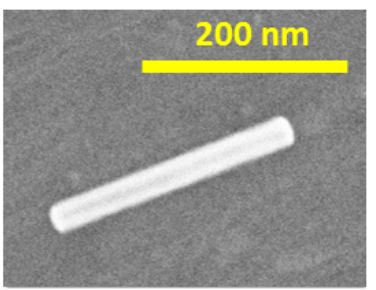

(e)

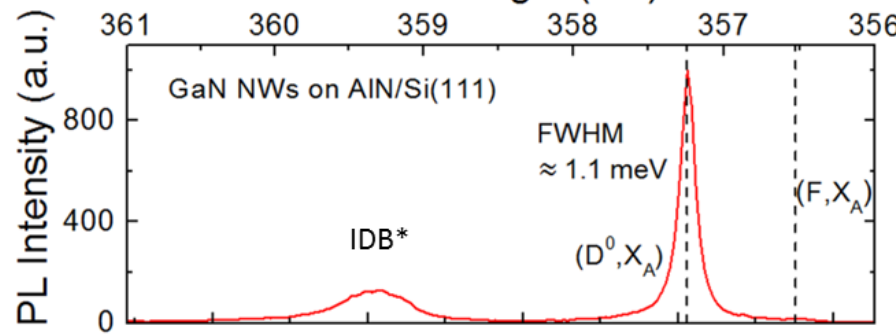

(f)

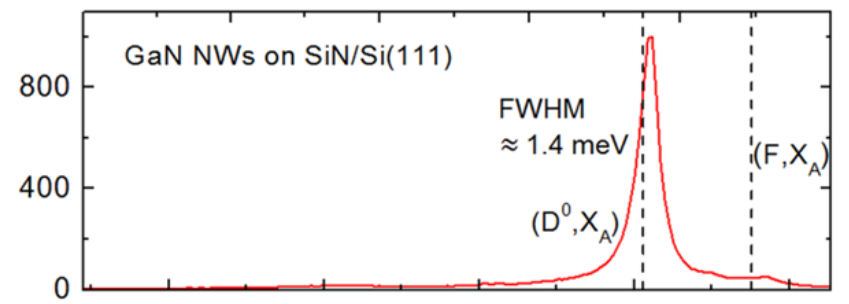

(g)

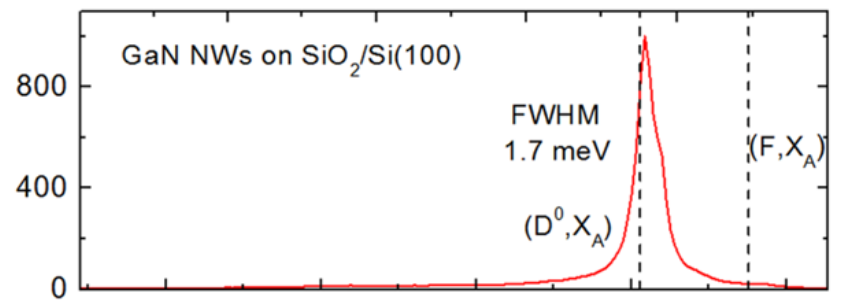

(h)

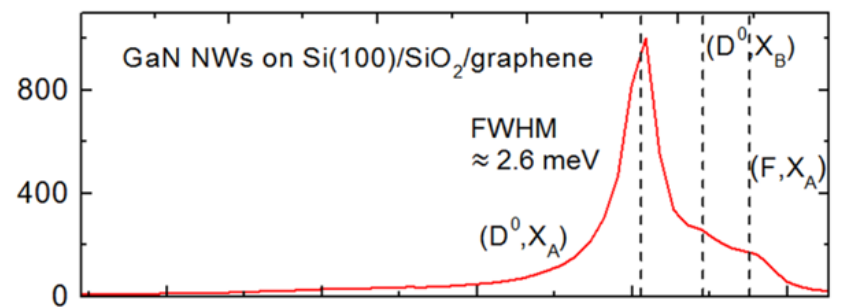

(j)

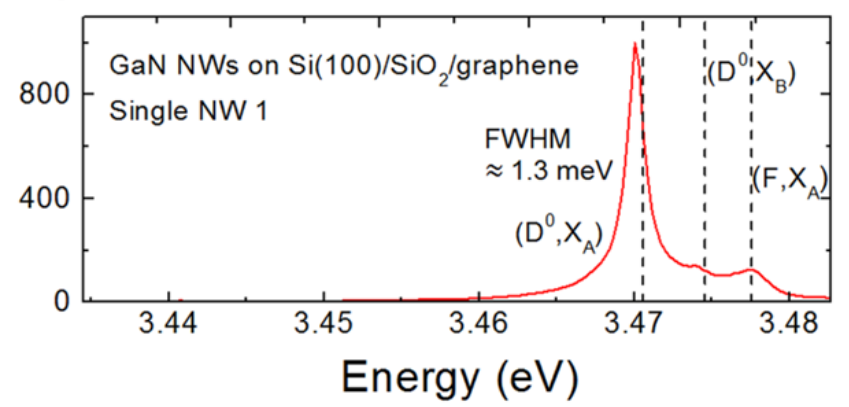

Fig 1. 45 -tilted SEM images and corresponding low temperature $\mu \mathrm{PL}$ spectra of GaN NWs grown on (a,e) AlN/Si(111), (b,f) SiN/Si(111), (c,g) $\mathrm{SiO}_{2} / \mathrm{Si}(100)$ and (d,h) graphene $/ \mathrm{SiO}_{2} / \mathrm{Si}(100)$. (i, j) Top view SEM image and $\mu \mathrm{PL}$ spectrum of a single GaN NW dispersed on $\mathrm{SiO}_{2}$ from the sample shown in panel (d).

To further analyse the optical properties of GaN NWs grown on graphene, the recombination dynamics was measured using time resolved PL (TR-PL). The description of the experimental setup and details about the experimental conditions under which TR-PL acquisitions were taken can be found in the methods section. 
For GaN NWs grown by PA-MBE on conventional substrates, several TR-PL analyses have been reported. Calleja et al. ${ }^{24}$ measured low temperature decay times of 170 and 240 ps for emissions around 3.47 and $3.45 \mathrm{eV}$, respectively. Schlager et al. ${ }^{36}$ reported excitons lifetimes of around 550 ps for both strained and relaxed structures (for considerably bigger structures and higher excitation powers) . Corfdir et al. ${ }^{23}$ measured typical lifetimes between 150 and $200 \mathrm{ps}$ for the donor bound exciton peak $(3.47 \mathrm{eV})$ and 320 and 470 ps for the $3.45 \mathrm{eV}$ peak. Gorgis et al. ${ }^{37}$ reported single exponential decay behaviour of the excitonic emission up to $30 \mathrm{~K}$ for single free-standing NWs. Individual decay times ranging from 50 to 300 ps were observed. Moreover, they observed nonexponential TR-PL transients for NW ensembles, which were ascribed to the convolution of single exponential decays with different decay times. Hauswald et al. ${ }^{38}$ demonstrated that the biexponential decays do not originate from spectral superposition of different states or from the NW surface, but the coupling between all exciton states participating in recombination determines their temporal evolution. A variety of GaN NWs were investigated by Hauswald et al.,with lifetimes ranging between 120 and $210 \mathrm{ps}$ for $\mathrm{NWs}$ on $\mathrm{Si}^{39}$. On TiN, Wölzand collaborators observed a lifetime of $230 \mathrm{ps}^{40}$.

Fig. 3 reports the low temperature $(10 \mathrm{~K})$ TR-PL decay traces acquired from self-assembled GaN $\mathrm{NW}$ arrays grown on graphene/ $\mathrm{SiO}_{2} / \mathrm{Si}(100)$ substrates (blue symbols). As a reference, TR-PL traces for NWs grown on AlN/Si(111) substrates are also shown (red symbols). Fig. 3(a) and (b) show the time dependency of the PL signals corresponding to $\left(\mathrm{D}^{0}, \mathrm{X}_{\mathrm{A}}\right)$ and $\left(\mathrm{F}, \mathrm{X}_{\mathrm{A}}\right)$ recombination processes, respectively. Single exponential fits of the data are reported for all curves (in full line for grown-on-AlN NWs and dashed lines for grown-on-graphene NWs). The resulting decay times for donor-bound exciton recombination processes are $105 \mathrm{ps}$ and $130 \mathrm{ps}$ for NWs grown on AIN and on graphene, respectively. Free excitons decay with characteristic time constants of around 55 ps for grown-on-AlN NWs and of 45 ps for those grown on graphene.

Streak camera images (up) and corresponding transient spectra (bottom) from which decay times were obtained are reported for NWs grown on AlN (c) and graphene (d). In (c), both peaks corresponding to IDB and bond exciton recombination can be seen, while (d) shows only the latter. It should be noted that due to the low signal-to-noise ratio of the transient spectra it is difficult to address the dynamics of the $\left(\mathrm{D}^{0}, \mathrm{X}_{\mathrm{A}}\right)$ and $\left(\mathrm{F}, \mathrm{X}_{\mathrm{A}}\right)$ processes separately. Exact peak positions for $\mathrm{PL}$ transients reported in (a) and (b) were inferred by steady state $\mu \mathrm{PL}$.

The results of the TR-PL analysis point out that the temporal dynamics of recombination processes in GaN NWs grown on graphene and on AlN substrates shows no substantial difference For NWs grown on $\mathrm{AlN} / \mathrm{Si}$, the decay time of the IDB emission was also assessed ( $300 \mathrm{ps}$, not shown here). This slower dynamics with respect to free- and donor-bound exciton recombination is consistent with the previous reports for GaN NWs ${ }^{23}$. 

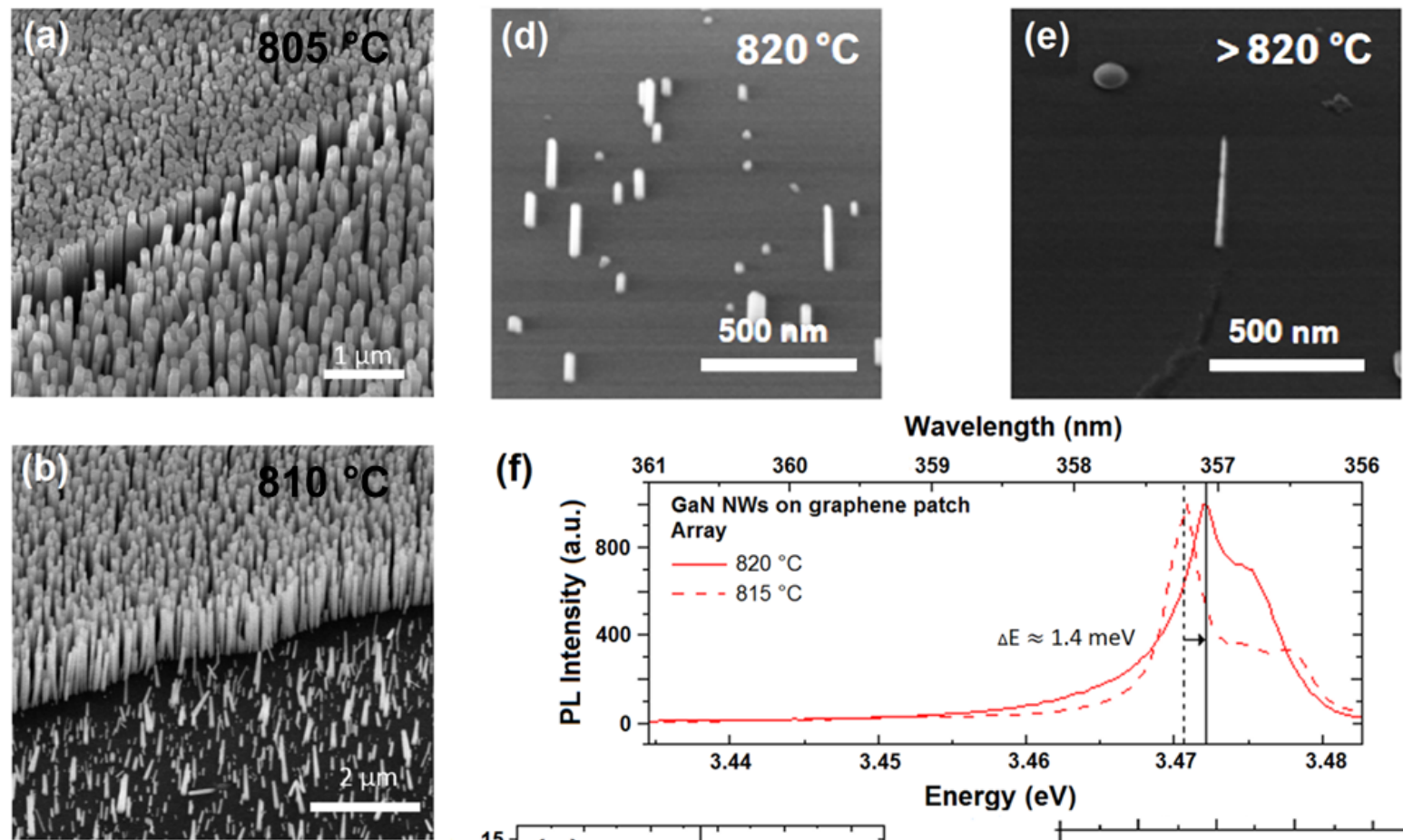

(f)
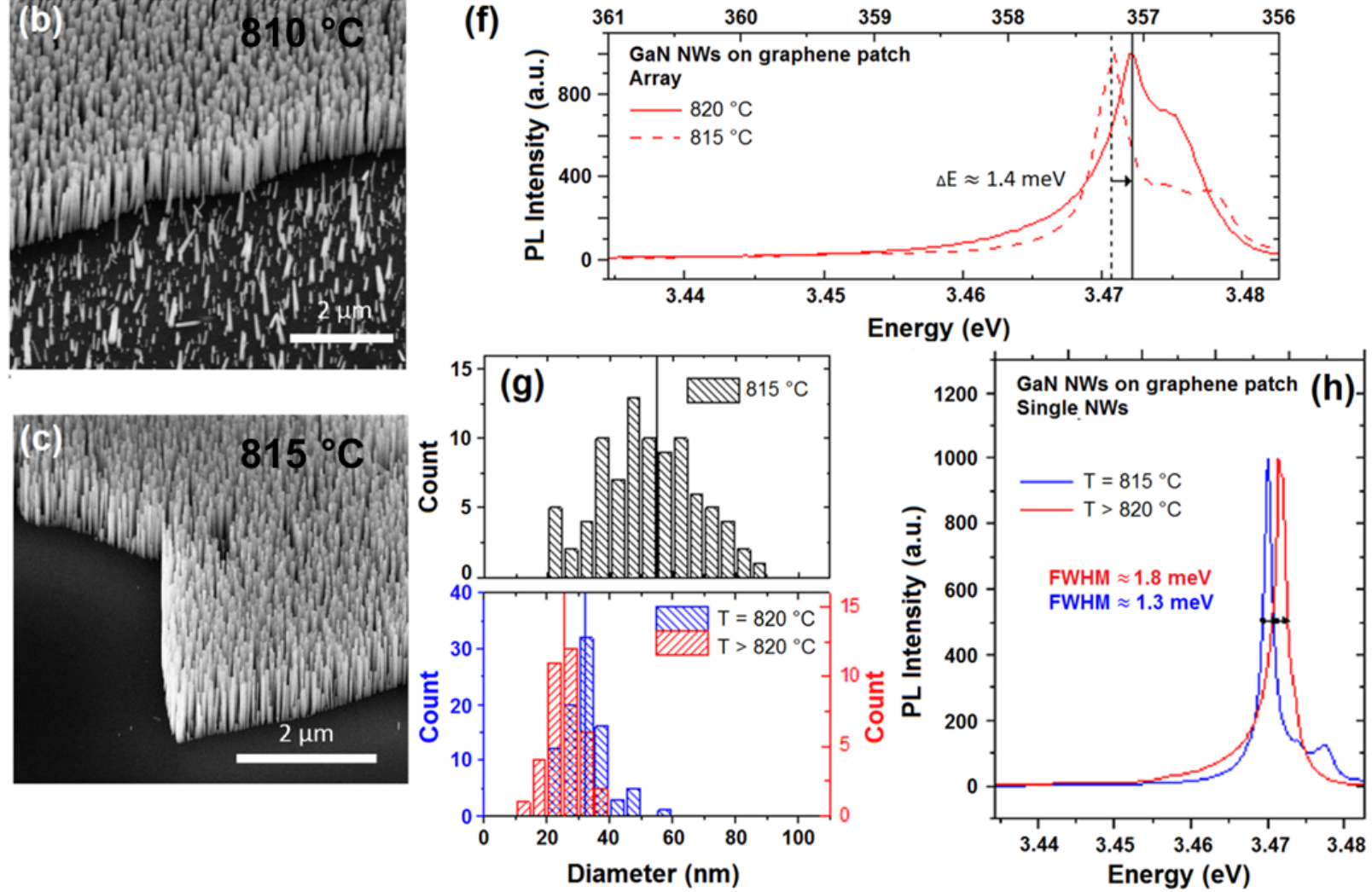

Fig.2 SEM images taken at $45^{\circ}$ of GaN NWs grown on graphene at (a) $805^{\circ} \mathrm{C}$, (b) $810^{\circ} \mathrm{C}$, (c) $815^{\circ} \mathrm{C}$, (d) $820^{\circ} \mathrm{C}$. (e) corresponds to a region of sample (d) where the temperature was higher than $820^{\circ} \mathrm{C}$. (f) $\mu \mathrm{PL}$ spectra of self-assembled GaN NWs grown on graphene at (full line) $820^{\circ} \mathrm{C}$ and (dashed line) $815^{\circ} \mathrm{C} .(\mathrm{g})$ Distributions of the measured NW diameters for samples grown at (black columns) $815^{\circ} \mathrm{C}$, (blue columns) $820^{\circ} \mathrm{C}$ and (red columns) at a temperature higher than $820^{\circ} \mathrm{C}$. The mean value of each distribution is indicated by a vertical line. (h) $\mu$ PL spectrum of single standing NWs grown at a temperature higher than $820^{\circ} \mathrm{C}$ (red line) and at $815^{\circ} \mathrm{C}$ (blue line).

In summary, the reported steady state $\mu \mathrm{PL}$ and TR-PL results confirm that the optical quality of self-assembled GaN NWs grown on CVD-graphene transferred on a $\mathrm{SiO}_{2}$ support is within the range of values reported in the literature for conventional substrates and is suitable for future photonic applications. 


\section{Influence of the growth temperature on the optical properties}

The optical properties of samples grown at temperatures above the optimal value were analysed as well. As previously discussed and illustrated in Fig. 2 (a) - (e), the temperature range within which dense arrays of self-assembled NWs can be selectively grown on graphene was observed to be relatively narrow. Panels (d) and (e) of Fig. 2 are taken from two different regions of the same sample, for which a temperature gradient of approximately $5{ }^{\circ} \mathrm{C}$ (from $820^{\circ} \mathrm{C}$ in the centre to $825^{\circ} \mathrm{C}$ at the edge) was estimated and exploited to explore the influence of a small temperature shift in a single growth run. At around $820{ }^{\circ} \mathrm{C}$ the selectivity on graphene still holds but the density of the NW ensemble is considerably lower (few NWs per $\mu \mathrm{m}^{2}$ ). The decrease of the density is accompanied by a reduction of the average wire diameter (see Fig. 2 g)). Increasing the temperature above $820{ }^{\circ} \mathrm{C}$, the NW density monotonically decreases and eventually the growth is completely inhibited. Fig. 2 (f) shows the $\mu \mathrm{PL}$ spectrum acquired from a sparse NW array grown at $820{ }^{\circ} \mathrm{C}$ (full red line) which is compared to a spectrum from an array grown at the optimal selectivity temperature $\left(815^{\circ} \mathrm{C}\right)$. The NBE emission is still dominated by donor-bound exciton recombination, but the $\left(\mathrm{D}^{0}, \mathrm{X}_{\mathrm{A}}\right)$ peak is blue-shifted by $1.4 \mathrm{meV}$ and broadened with respect to the emission of NWs grown at $815^{\circ} \mathrm{C}$. Both effects can be ascribed to the $\mathrm{NW}$ morphology dependence on growth temperature. Fig. 2 (g) reports the statistical distribution of the diameters of NWs grown at $815^{\circ} \mathrm{C}$ (black bars), $820{ }^{\circ} \mathrm{C}$ (blue bars) and higher than $820{ }^{\circ} \mathrm{C}$ (red bars) temperatures. For each distribution the average diameter is highlighted by a vertical line. When the temperature is increased from 815 to $820{ }^{\circ} \mathrm{C}$, the average diameter decreases from 55 to $30 \mathrm{~nm}$. For higher temperatures close to the edge, the average of the distribution goes towards even lower values (average diameter of $25 \mathrm{~nm}$ in the lowest density region is observed).

For such small diameters, the transition energy can differ from the bulk due to two effects. First, the dielectric confinement induced by a mismatch in dielectric constants between $\mathrm{GaN}$ and vacuum may blue-shift the transition energy as reported in ${ }^{41}$. Second, surface stress (analogous to surface tension and the resulting Laplace pressure for liquids) can exert a strain and thus induce a blueshift of the emission [G. Calabrese, V.M. Kaganer, O. Brandt, unpublished]. Since the transition energy is now sensitive to fluctuations of the NWdiameter, the emission from the NW array broadens ${ }^{41,42}$. Nevertheless, we could find isolated single NWs whose low temperature $\mu \mathrm{PL}$ emission is characterized by a relatively sharp $\left(\mathrm{D}^{0}, \mathrm{X}_{\mathrm{A}}\right)$ peak. The $\mu \mathrm{PL}$ spectrum of such a NW (Fig.3 (h)) shows a linewidth of about $1.8 \mathrm{meV}$. 

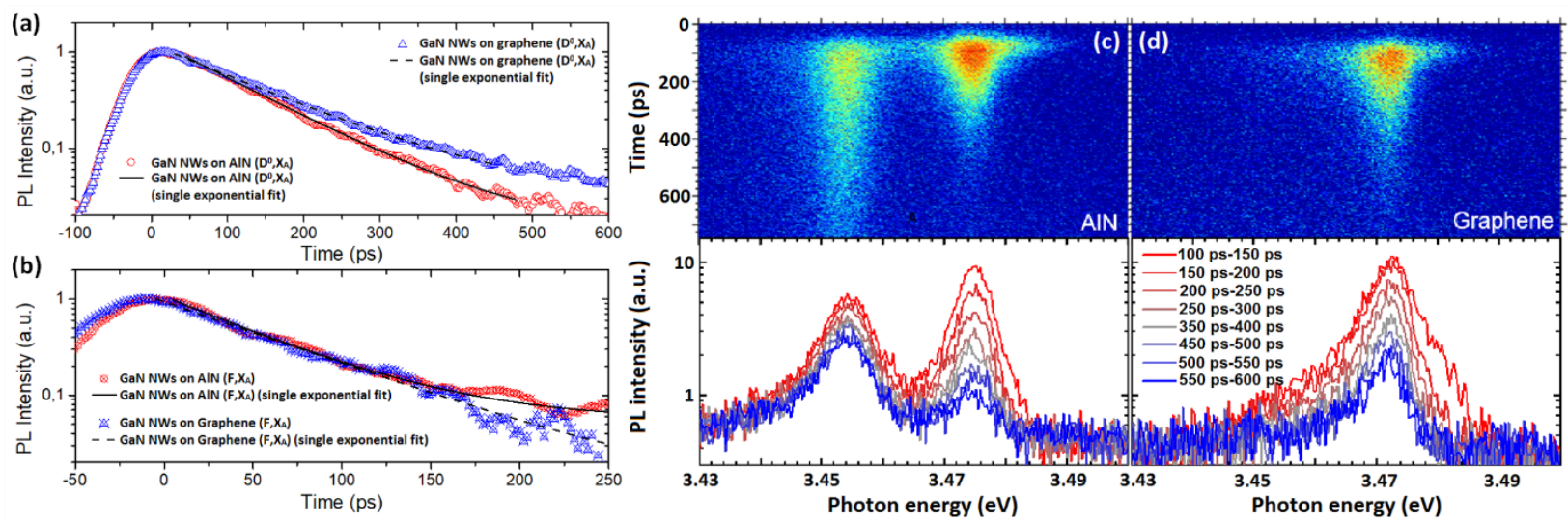

Fig.3 TR-PL decay traces of GaN NW ensembles grown on AlN/Si(111) (red symbols) and graphene $/ \mathrm{SiO}_{2} / \mathrm{Si}(100)$ (blue symbols) substrates. Temporal traces corresponding to (a) the $\left(\mathrm{D}^{0}, \mathrm{X}_{\mathrm{A}}\right)$ and (b) the $\left(\mathrm{F}, \mathrm{X}_{\mathrm{A}}\right)$ excitonic emissions are reported. The single exponential fit of each series of data is reported for NWs grow on $\mathrm{AlN} / \mathrm{Si}(111)$ (full line) and on graphene $/ \mathrm{SiO}_{2} / \mathrm{Si}(100)$ (dashed line).Streak camera images (up) and corresponding transient spectra (bottom) from which temporal traces in (a) and (b) were obtained are reported for NWs grown on AlN (c) and graphene (d).

\section{Optical properties of GaN nanowires on graphene micro-domains}

In the following the optical properties of a small number of standing NWs selectively grown on multi-layered graphene domains of $\mu \mathrm{m}$ and sub- $\mu \mathrm{m}$ sizes are described. Multi-layer graphene domains were grown on $\mathrm{Cu}$ foils by pulsed-CVD technique as described in Methods section. To obtain hexagonal multi-layer graphene domains, the growth was carried out on a defective $\mathrm{Cu}$ substrate ${ }^{43,44}$ and interrupted before the lateral coalescence of the domains. As a result, a mixture of mono-layered domains and multi-layered domains with inverted-ladder geometry is obtained. This bi-modal population is believed to depend on whether or not the graphene layer forms on a $\mathrm{Cu}$ defective site as schematically presented in Fig. 4 (a) illustrating the growth mechanism. Generally, after the catalytic decomposition of $\mathrm{CH}_{4}$ precursors on the $\mathrm{Cu}$ surface, $\mathrm{C}$ atoms diffuse and eventually rearrange in a $\mathrm{sp}^{2}$ hybridization (with no chemical bonding to the $\mathrm{Cu}$ surface). This process leads to the nucleation and lateral growth of graphene domains of strictly 1 monolayer (left side of Fig 4 (a) ${ }^{45}$. However, if the growth takes place in proximity of a defect, $\mathrm{C}$ atoms can diffuse within the $\mathrm{Cu}$ substrate, and when they re-emerge, they incur into subsequent nucleation and growth events. These processes lead to the growth of a multi-layered graphene domains ${ }^{43,44}$ (the number of layers in their central part roughly estimated by SEM contrast goes up to 8 MLs) with an inverted-ladder structure (right-side of Fig 4 (a)), i.e. with a number of layers which increases from the edge of the domain towards its center. Raman analysis confirmed the multi-layered nature of the domains as described in the methods section. Moreover, Raman spectroscopy was repeated after NW growth demonstrating that the signatures of a multilayered graphene were preserved. Therefore, graphene was not completely deteriorated by the nitrogen plasma ${ }^{32}$. The size of the domains is controlled by the growth time and can be reduced below $1 \mu \mathrm{m}$ (an example of a sub$\mu \mathrm{m}$ graphene domain on a copper foil in shown in Fig 4 (b). 
These graphene multi-layered domains were used to obtain GaN NWs arranged in a hollow hexagonal sub- $\mu \mathrm{m}$ pattern. Fig. 4 (g) shows a top view SEM image of a large sample area illustrating a typical morphology after the NW growth with few monolayer domains entirely filled with NWs and numerous hollow structures corresponding to multi-layered domains. Close-up SEM images of one hollow hexagonal pattern in top-view and $45^{\circ}$-tilted configurations are shown in panels (c) and (d), respectively. The existing relationship between the number of atomic layers composing the graphitic substrate and the density of the GaN NW array growing on top of it was previously established by Kumaresan et al. for large graphene domains of several tens of $\mu \mathrm{m}^{15}$. It was observed that the NW density decreases rapidly with the number of underlying graphene layers. Here, we report a similar observation for the $\mu \mathrm{m}$ and sub- $\mu \mathrm{m}$ domains: the increase of the number of graphene layers toward the centre of the inverted hexagonal pyramid leads to a ring of NWs growing at the periphery of the domain and no growth in the central region.
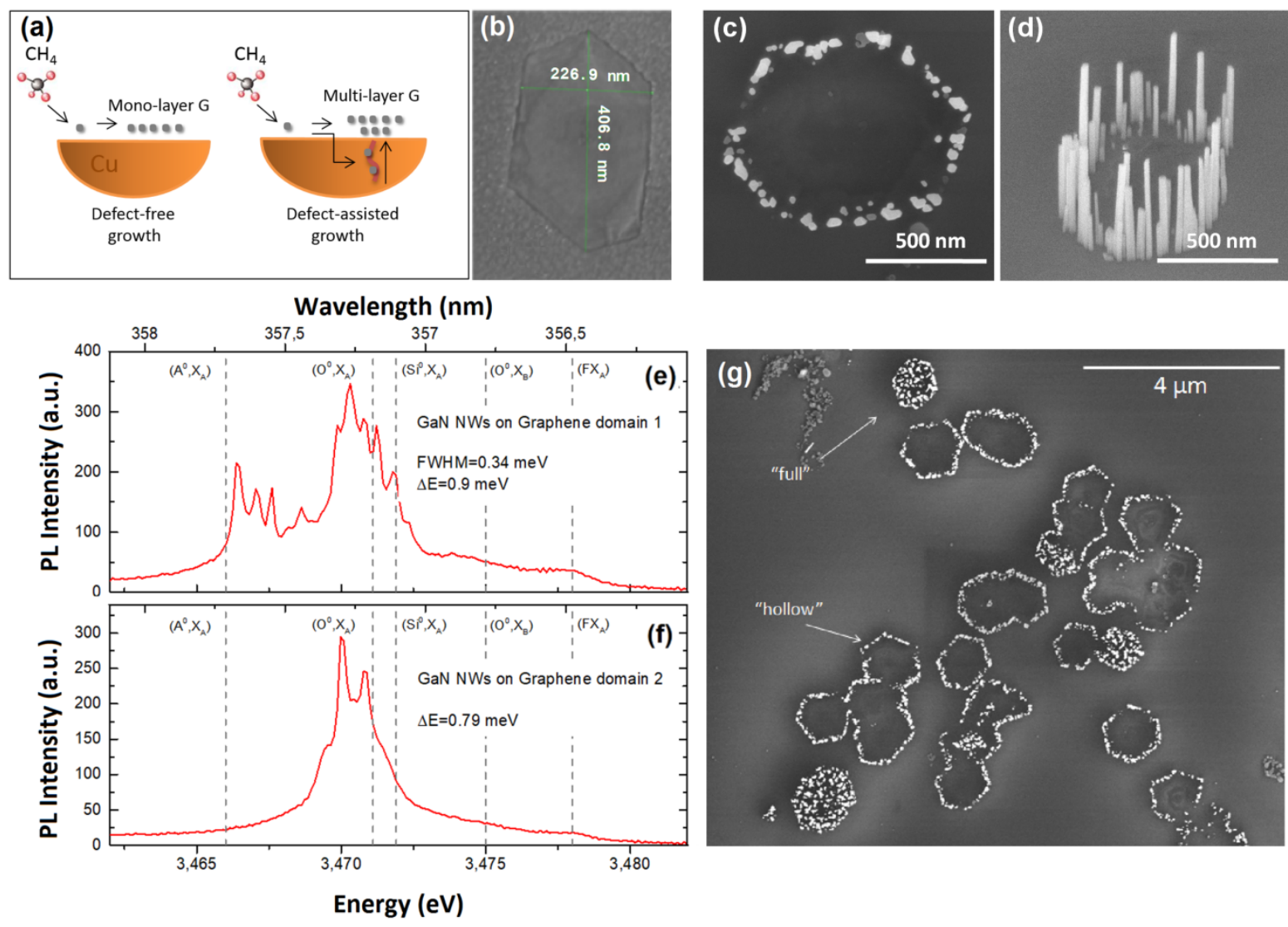

Fig. 4 (a) Schematic showing the defect-free and defect-assisted growth of monolayer and multi-layered graphene domains by CVD. (b) SEM image of one $200 \mathrm{~nm}$ sized graphene domain on Cu foil.Top-view (c) and (g) and $45^{\circ}$ tilted (d) SEM images of GaN NWs grown on microscopic graphene domains. (e,f) $\mu$ PL spectra of GaN NWs grown on two different domains. Energies corresponding to the different excitonic recombination processes are indicated by vertical dashed lines. 
The optical properties of GaN NWs grown on graphene micro-domains were assessed by $\mu \mathrm{PL}$ analysis. A different experimental setup was used to increase the spatial and spectral resolution, as described in details in the methods section. Note that the spatial resolution of about $0.3 \mu \mathrm{m}$ together with the peculiar circular arrangement of the NWs, results in spectra to which only a few NWs have contributed. Fig. 4 (e) and (f) report $\mu$ PL spectra acquired from two different hollow domains. Vertical dashed lines highlight the energy values corresponding to donor-bound $\left(\mathrm{O}^{0}, \mathrm{X}_{\mathrm{A}}\right),\left(\mathrm{Si}^{0}, \mathrm{X}_{\mathrm{A}}\right)$, $\left(\mathrm{O}^{0}, \mathrm{X}_{\mathrm{B}}\right)$, acceptor-bound $\left(\mathrm{A}^{0}, \mathrm{X}_{\mathrm{A}}\right)$ and free $\left(\mathrm{FX}_{\mathrm{A}}\right)$ exciton recombination processes for unstrained

bulk $\mathrm{GaN}^{46-48}$. The spectra consist of multiple narrow lines originating from a small number of NWs contained within a single graphene domain. The sharp peaks characterizing the spectra are identified by taking into account their relative energy differences: as previously observed for NW arrays, the emission is dominated by donor bound excitons, but in this case the higher spectral resolution ensured by the experimental setup and the relatively small number of excited NWs allow us to distinguishthe contributions from the oxygen and silicon donor-bound excitontransitions $\left(\mathrm{O}^{0}, \mathrm{X}_{\mathrm{A}}\right)$ and $\left(\mathrm{Si}^{0}, \mathrm{X}_{\mathrm{A}}\right)$, separated in energy by $0.8 \mathrm{meV}$. In Fig. 4 (e) a group of acceptor- and donorbound excitons lines as well as the free exciton bandcan clearly be identified. Both spectra reported in Fig.4 (e) and (f) show oxygen and silicon donor-bound excitonic transitions which are redshifted with respect to the energies of the corresponding transitions expected for unstrained GaN. The red-shift of these transition energies is likely due to the micro-strain introduced by the coalescence of adjacent $\mathrm{NWs}{ }^{22,28,49,50}$, which is seen to take place frequently at the periphery of the hollow domains (cf. Fig. 4(c)). It should be noted that the spectral linewidth of the peaks is close to the spectral resolution of $0.25 \mathrm{meV}$ and is comparable with the best results for single NWs and NW bunches grown on conventional substrates ${ }^{26,30}$.

\section{Conclusions}

In conclusion, we have analysed the optical properties of GaN NWs grown by PA-MBE on CVDgraphene transferred onto $\mathrm{SiO}_{2}$. We compared them with the properties of NWs grown on other crystalline and amorphous substrates. The influence of substrate temperature on the selectivity of growth on the $\mathrm{SiO}_{2}$ surface or on graphene was investigated. A high density of GaN NWs can be selectively grown on graphene in a narrow temperature window centred around $815{ }^{\circ} \mathrm{C}$. The morphology and the optical quality of self-assembled NWs on graphene macro-patches are comparable to those of NWs grown on other substrates. Their optical signature is dominated by the donor-bound excitons $\left(\mathrm{D}^{0}, \mathrm{X}_{\mathrm{A}}\right)$. However, GaN NWs grown on graphene patches and microdomains showed no emission associated with IDBs. The influence of growth temperature on the morphology and optical properties of NWs was discussed. In particular, the emission from single free-standing NWs grown above $820{ }^{\circ} \mathrm{C}$ was characterized by donor-bound exciton transitions blue-shifted due to the diameter reduction and dielectric confinement effects. $\mu \mathrm{m}$ and sub- $\mu \mathrm{m}$ graphene domains were proposed as growth templates for obtaining novel sub- $\mu \mathrm{m}$ hollow hexagonal motifs of vertical GaN NWs. These NWs are characterized by a spectral linewidth as low as the spectral resolution of $0.25 \mathrm{MeV}$ for the dominant donor-bound exciton transition.

\section{Methods:}


Graphene growth and transfer.Commercial monolayer polycrystalline (grain size up to $20 \mu \mathrm{m}$ ) graphene patches grown on $\mathrm{Cu}$ foils by CVD were purchased from Graphenea supermarket. Multilayered graphene domains were home-grown on $\mathrm{Cu}$ foils by pulsed-CVD technique. The experimental conditions were: a gas pressure of $25 \mathrm{mbar}$, a growth temperature of $1000^{\circ} \mathrm{C}$ and ten cycles of $\mathrm{CH}_{4}(20 \mathrm{sccm}, 5 \mathrm{~s})$ and $\mathrm{H}_{2}(960 \mathrm{sccm}, 55 \mathrm{~s})$. Both monolayer graphene patches and graphene micro-domains were transferred to $\mathrm{SiO}_{2}(300 \mathrm{~nm}) / \mathrm{Si}$ substrates following the procedure described by X. Li at al ${ }^{51}$ In a first step, the CVD graphene-on-copper foil was spin-coated with a $\sim 50 \mathrm{~nm}$-thick poly(methyl methacrylate) (PMMA) layer. Then, the copper foil was etched within a $\left(\mathrm{NH}_{4}\right)_{2} \mathrm{~S}_{2} \mathrm{O}_{8}$ bath solution, leaving a floating graphene/PMMA layer on the liquid surface ${ }^{52}$. This was rinsed into multiple deionized water baths, and finally fished with the $300 \mathrm{~nm} \mathrm{SiO}_{2}$ on-Si(100) substrate, which was dried in ambient atmosphere. Finally, the PMMA protective layer was removed by solvent cleaning (acetone, isopropyl alcohol) ${ }^{53,54}$, and the substrate was introduced into vacuum within few days from its preparation for a better conservation before the NW growth.

Steady state $\mu P L$. All NW samples were analyzed at $5 \mathrm{~K}$ in a continuous flow He cryostat. Excitation was provided through an objective with a numerical aperture NA $=0.4$ by a continuous $244 \mathrm{~nm} \mathrm{Ar}{ }^{++}$laser with a spot size of around $1 \mu \mathrm{m}$. Laser incident powers of around $1 \mu \mathrm{W}$ and 100 $\mu \mathrm{W}$ were used for NW arrays and for single dispersed NWs, respectively. The generated PL signal was dispersed by a 1200 lines $/ \mathrm{mm}$ grating in a $460 \mathrm{~mm}$ focal length spectrometer allowing for a spectral resolution of around $0.5 \mathrm{meV}$ and collected by a $\mathrm{LN}_{2}$-cooled silicon charge-coupled device (CCD).

GaN NWs grown on graphene domains were further analyzed using a $\mu \mathrm{PL}$ setup with a higher resolution. Samples were cooled down to $10 \mathrm{~K}$. The measurement was done under continuous 325 $\mathrm{nm}$ laser excitation through a $\times 50$ objective with $\mathrm{NA}=0.65$, providing an excitation density going from $900 \mathrm{~W} / \mathrm{cm}^{2}$ to $9 \mathrm{~kW} / \mathrm{cm}^{2}$. The emitted signal was dispersed by 2400 lines $/ \mathrm{mm}$ spectrometer grating allowing for a spectral resolution of $0.25 \mathrm{meV}$ within the considered spectral range.

Time resolved-PL. A closed-cycle allowed for controlling the sample temperature within the 10$300 \mathrm{~K}$ range. A Ti-sapphire laser with third harmonic generation $(266 \mathrm{~nm}, \mathrm{FWHM}=200 \mathrm{fs}$, repetition rate $=4 \mathrm{MHz}$ ) provided the optical excitation through a microscope objective lens with long working distance $(\times 80$, N.A. $=0.55)$. The emitted signal was dispersed on a spectrometer grating of 1800 lines $/ \mathrm{mm}$ and collected by CCD. The system's spectral resolution is $\sim 0.23 \mathrm{meV}$. A streak camera allowing a temporal resolution of around 8 ps was used for studying the dynamics of the PL emission.

Raman Spectroscopy. Raman spectra were measured using a inVia Micro Raman spectrometer, equipped with a frequency-doubled Neodymium-doped yttrium aluminum garnet laser operating at $532 \mathrm{~nm}$. The acquisitions are performed in back-scattering configuration, through a $\times 100$ objective, yielding a spot-size fixed by the diffraction limit of $300 \mathrm{~nm}$. The signal is dispersed by a 1800 lines/mm grating allowing spectral resolution of $0.40 \mathrm{~cm}^{-1}$ for $10 \mathrm{sec}$ acquisition time, and collected by a photodiode. Fig. 5 displays Raman spectra normalized with respect to the peak at 
around $1580 \mathrm{~cm}^{-1}$ (G peak) of a commercial $1 \mathrm{~cm}^{2}$ graphene patch with an average thickness of 1 ML (black line), of a sub-micrometer graphene domain sample before the NW growth (red line) and after the growth (blue line). As previously reported ${ }^{55,56}$, for multi-layered graphene, because of added forces from the interactions between layers (AB-stacked), as the number of graphene layers increases, the second order 2D peak splits in an increasing number of modes (few-Lorentzian 2D peaks), resulting in a wider, shorter, higher frequency peak. It follows that the number of layers can potentially be derived from the ratio of peak intensities, $\mathrm{I}_{2 \mathrm{D}} / \mathrm{I}_{\mathrm{G}}$, as well as from their position and shape ${ }^{57}$. However, due to possible difference in doping level, it is difficult to quantitatively estimate the number of layers by Raman technique ${ }^{58}$. Despite the limited spatial resolution of the technique not allowing to resolve the ladder-like structure at the edges of the hexagonal domains, the red-shifted $\left(\sim 40 \mathrm{~cm}^{-1}\right)$, large (FWHM $\sim 80 \mathrm{~cm}^{-1}$ ) and low intensity 2D peaks observed on several domains before the NW growth and on "hollow" domains after the growth (red and blue curves on Fig. 5), clearly indicate a multi-layered stacking for these structures ${ }^{59}$. The Raman spectroscopy analysis thus confirms the multi-layered nature of the domains and suggests that the growth of graphene domains is characterized by a truncated hexagonal pyramid thickness profile, which inhibits the NW growth within the thicker central region of the domain while allowing them to grow only on the perimeter of the hexagonal pyramid base. The D peak intensity is relatively weak in both cases (before and after growth), which indicates a good structural quality of the graphene. The comparison of the D peak intensity of the Raman spectra before and after the NW growth suggests that the damage which can be expected from the nitrogen plasma ${ }^{32}$ was not dramatic and the multi-layered nature of the domains was preserved after the growth.

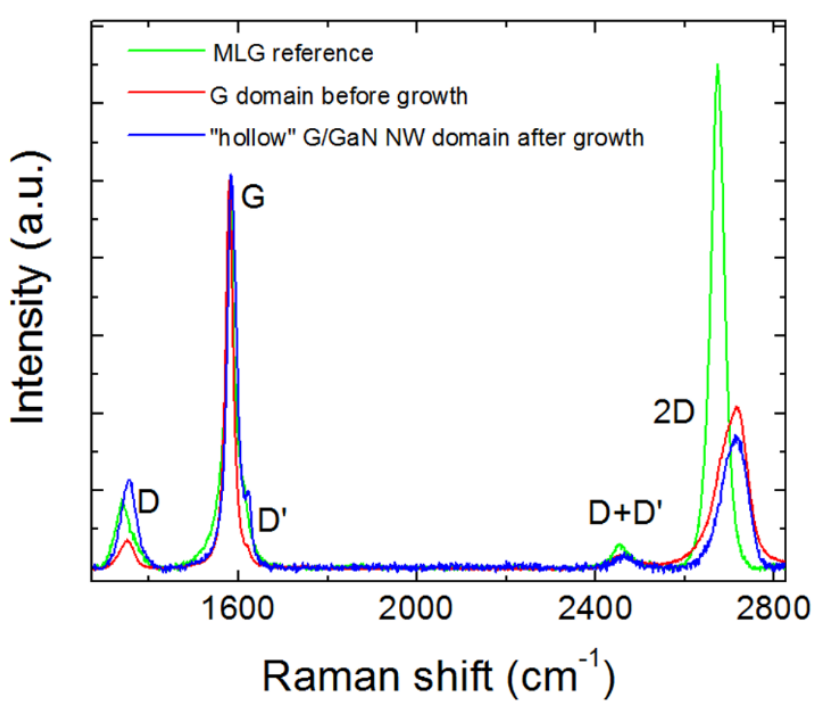

Fig. 5 Raman spectra normalized with respect to the G peak, of a commercial 1 ML graphene patch (green line), of a sub-micrometer graphene domain before the NWs growth (red) and after the growth (blue). 


\section{Acknowledgments:}

This work has been financially supported by EU H2020 ERC project "NanoHarvest" (grant no. 639052), and French national LabexGaNex (ANR-11-LABX-2014).

This work has been supported by the Paternariat Hubert Curien between France and South Korea, project "STAR" (ref: 36626UF).

A. Babichev acknowledges the support of the Russian ScienceFoundation (project no. 18-7200157).

\section{References}

1. Koma, A., Sunouchi, K. \& Miyajima, T. Fabrication and characterization of heterostructures with subnanometer thickness. Microelectronic Engineering2, 129-136 (1984).

2. Hong, Y. J. \& Lee, C.-H. Chapter Three - van der Waals Heteroepitaxy of Semiconductor Nanowires. in Semiconductors and Semimetals (eds. Morral, A. F. I., Dayeh, S. A. \& Jagadish, C.) 93, 125-172 (Elsevier, 2015).

3. Tran, C. A. et al.High brightness GaN vertical light emitting diodes on metal alloyed substrate for general lighting application. Journal of Crystal Growth298, 722-724 (2007).

4. Guan, N., Dai, X., Babichev, A. V., Julien, F. H. \& Tchernycheva, M. Flexible inorganic light emitting diodes based on semiconductor nanowires. Chem. Sci.8, 7904-7911 (2017).

5. Kim, J. et al. Principle of direct van der Waals epitaxy of single-crystalline films on epitaxial graphene. Nature Communications5, 4836 (2014).

6. Kobayashi, Y., Kumakura, K., Akasaka, T. \& Makimoto, T. Layered boron nitride as a release layer for mechanical transfer of GaN-based devices. Nature484, 223-227 (2012).

7. Hiroki, M. et al. Suppression of self-heating effect in AIGaN/GaN high electron mobility transistors by substrate-transfer technology using h-BN. Appl. Phys. Lett.105, 193509 (2014). 
8. Ayari, T. et al. Wafer-scale controlled exfoliation of metal organic vapor phase epitaxy grown InGaN/GaN multi quantum well structures using low-tack two-dimensional layered h-BN. Appl. Phys. Lett.108, 171106 (2016).

9. Paduano, Q., Snure, M., Siegel, G., Thomson, D. \& Look, D. Growth and characteristics of AlGaN/GaN heterostructures on sp2-bonded BN by metal-organic chemical vapor deposition. Journal of Materials Research31, 2204-2213 (2016).

10. Li, X. et al. Flexible metal-semiconductor-metal device prototype on wafer-scale thick boron nitride layers grown by MOVPE. Scientific Reports7, 786 (2017).

11. Chung, K. et al. Growth and characterizations of GaN micro-rods on graphene films for flexible light emitting diodes. APL Materials2, 092512 (2014).

12. Kang, S. et al. Ultraviolet photoconductive devices with an n-GaN nanorod-graphene hybrid structure synthesized by metal-organic chemical vapor deposition. Scientific Reports5, 10808 (2015).

13. Heilmann, M. et al. Vertically Oriented Growth of GaN Nanorods on Si Using Graphene as an Atomically Thin Buffer Layer. Nano Lett.16, 3524-3532 (2016).

14. Heilmann, M. et al. Growth of GaN Micro- and Nanorods on Graphene-Covered Sapphire: Enabling Conductivity to Semiconductor Nanostructures on Insulating Substrates. Crystal Growth \& Design15, 2079-2086 (2015).

15. Kumaresan, V. et al. Epitaxy of GaN Nanowires on Graphene. Nano Lett.16, 4895-4902 (2016).

16. Hayashi, H., Konno, Y. \& Kishino, K. Self-organization of dislocation-free, high-density, vertically aligned GaN nanocolumns involving InGaN quantum wells on graphene/SiO 2 covered with a thin AIN buffer layer. Nanotechnology27, 055302 (2016).

17. Araki, T. et al. Radio-frequency plasma-excited molecular beam epitaxy growth of GaN on graphene/Si(100) substrates. Appl. Phys. Express7, 071001 (2014). 
18. Nakagawa, S., Tabata, T., Honda, Y., Yamaguchi, M. \& Amano, H. GaN Nanowires Grown on a Graphite Substrate by Radio Frequency Molecular Beam Epitaxy. Jpn. J. Appl. Phys.52, 08JE07 (2013).

19. Fernández-Garrido, S., Zettler, J. K., Geelhaar, L. \& Brandt, O. Monitoring the Formation of Nanowires by Line-of-Sight Quadrupole Mass Spectrometry: A Comprehensive Description of the Temporal Evolution of GaN Nanowire Ensembles. Nano Lett.15, 1930-1937 (2015).

20. Park, T. S., Chung, C. I., Jung, S. M. \& Jeon, D. Z. FIM, RHEED STUDIES ON ATOMATIC STRUCTURE OF Si(111) AND Si(111)-Ag SURFACES. J. Phys. Colloques49, C6-275-C6-280 (1988).

21. Sobanska, M., Dubrovskii, V. G., Tchutchulashvili, G., Klosek, K. \& Zytkiewicz, Z. R. Analysis of Incubation Times for the Self-Induced Formation of GaN Nanowires: Influence of the Substrate on the Nucleation Mechanism. Crystal Growth \& Design16, 7205-7211 (2016).

22. Robins, L. H., Bertness, K. A., Barker, J. M., Sanford, N. A. \& Schlager, J. B. Optical and structural study of GaN nanowires grown by catalyst-free molecular beam epitaxy. I. Near-band-edge luminescence and strain effects. Journal of Applied Physics101, 113505 (2007).

23. Corfdir, P. et al. Time-resolved spectroscopy on GaN nanocolumns grown by plasma assisted molecular beam epitaxy on Si substrates. Journal of Applied Physics105, 013113 (2009).

24. Calleja, E. et al. Luminescence properties and defects in GaN nanocolumns grown by molecular beam epitaxy. Phys. Rev. B62, 16826-16834 (2000).

25. Van Nostrand, J. E. et al. Molecular beam epitaxial growth of high-quality GaN nanocolumns. Journal of Crystal Growth287, 500-503 (2006).

26. Brandt, O., Pfüller, C., Chèze, C., Geelhaar, L. \& Riechert, H. Sub-meV linewidth of excitonic luminescence in single GaN nanowires: Direct evidence for surface excitons. Phys. Rev. B81, 045302 (2010).

27. Pfüller, C. et al. Statistical analysis of excitonic transitions in single, free-standing GaN nanowires: Probing impurity incorporation in the poissonian limit. Nano Res.3, 881-888 (2010). 
28. Jenichen, B. et al. Macro- and micro-strain in GaN nanowires on Si(111). Nanotechnology22, 295714 (2011).

29. Corfdir, P. et al. Sub-meV linewidth in GaN nanowire ensembles: Absence of surface excitons due to the field ionization of donors. Phys. Rev. B90, 205301 (2014).

30. Corfdir, P., Feix, F., Zettler, J. K., Fernández-Garrido, S. \& Brandt, O. Importance of the dielectric contrast for the polarization of excitonic transitions in single GaN nanowires. New J. Phys.17, 033040 (2015).

31. Zettler, J. K. et al. Improved control over spontaneously formed GaN nanowires in molecular beam epitaxy using a two-step growth process. Nanotechnology26, 445604 (2015).

32. Fernández-Garrido, S. et al. Molecular Beam Epitaxy of GaN Nanowires on Epitaxial Graphene. Nano Lett.17, 5213-5221 (2017).

33. Auzelle, T. et al. Attribution of the $3.45 \mathrm{eV} \mathrm{GaN}$ nanowires luminescence to inversion domain boundaries. Appl. Phys. Lett.107, 051904 (2015).

34. Pfüller, C. et al. Nature of excitons bound to inversion domain boundaries: Origin of the 3.45-eV luminescence lines in spontaneously formed GaN nanowires on Si(111). Phys. Rev. B94, 155308 (2016).

35. Dimitrakopulos, G. P. et al. Interfacial steps, dislocations, and inversion domain boundaries in the GaN/AIN/Si (0001)/(111) epitaxial system. physica status solidi (b)242, 1617-1627 (2005).

36. Schlager, J. B. et al.Steady-state and time-resolved photoluminescence from relaxed and strained GaN nanowires grown by catalyst-free molecular-beam epitaxy. Journal of Applied Physics103, 124309 (2008).

37. Gorgis, A. et al. Time-resolved photoluminescence spectroscopy of individual GaN nanowires. Phys. Rev. B86, 041302 (2012). 
38. Hauswald, C. et al. Coupling of exciton states as the origin of their biexponential decay dynamics in GaN nanowires. Physical Review B88, (2013).

39. Hauswald, C. et al. Origin of the nonradiative decay of bound excitons in GaN nanowires. Phys. Rev. B90, 165304 (2014).

40. Wölz, M. et al. Epitaxial Growth of GaN Nanowires with High Structural Perfection on a Metallic TiN Film. Nano Lett.15, 3743-3747 (2015).

41. Zettler, J. K. et al. Observation of Dielectrically Confined Excitons in Ultrathin GaN Nanowires up to Room Temperature. Nano Letters16, 973-980 (2016).

42. Corfdir, P. et al. Crystal-phase quantum dots in GaN quantum wires. Phys. Rev. B93, 115305 (2016).

43. Su, C.-Y. et al. Direct Formation of Wafer Scale Graphene Thin Layers on Insulating Substrates by Chemical Vapor Deposition. Nano Lett.11, 3612-3616 (2011).

44. Han, G. H. et al. Influence of Copper Morphology in Forming Nucleation Seeds for Graphene Growth. Nano Lett.11, 4144-4148 (2011).

45. Hu, B. et al. Epitaxial growth of large-area single-layer graphene over $\mathrm{Cu}(111) /$ sapphire by atmospheric pressure CVD. Carbon50, 57-65 (2012).

46. Monemar, B. Bound excitons in GaN. J. Phys.: Condens. Matter13, 7011 (2001).

47. Monemar, B. Fundamental energy gap of GaN from photoluminescence excitation spectra. Phys. Rev. B10, 676-681 (1974).

48. Kornitzer, K. et al. Photoluminescence and reflectance spectroscopy of excitonic transitions in high-quality homoepitaxial GaN films. Phys. Rev. B60, 1471-1473 (1999).

49. Brandt, O. et al. Statistical Analysis of the Shape of One-Dimensional Nanostructures: Determining the Coalescence Degree of Spontaneously Formed GaN Nanowires. Crystal Growth \& Design14, 2246-2253 (2014). 
50. Kaganer, V. M. et al. Inhomogeneous strain in GaN nanowires determined from x-ray diffraction peak profiles. Phys. Rev. B86, 115325 (2012).

51. Li, X. et al. Large-Area Synthesis of High-Quality and Uniform Graphene Films on Copper Foils. Science324, 1312-1314 (2009).

52. Babichev, A. V. et al. Influence of Substrate Microstructure on the Transport Properties of CVDGraphene. ACS Appl. Mater. Interfaces8, 240-246 (2016).

53. Tchernycheva, M. et al. Integrated Photonic Platform Based on InGaN/GaN Nanowire Emitters and Detectors. Nano Lett.14, 3515-3520 (2014).

54. Babichev, A. V., Gasumyants, V. E., Egorov, A. Y., Vitusevich, S. \& Tchernycheva, M. Contact properties to CVD-graphene on GaAs substrates for optoelectronic applications. Nanotechnology25, 335707 (2014).

55. Ferrari, A. C. et al. Raman Spectrum of Graphene and Graphene Layers. Phys. Rev. Lett.97, 187401 (2006).

56. O'Brien, M. \& Nichols, B. CVD Synthesis and Characterization of Graphene Thin Films. (ARMY RESEARCH LAB ADELPHI MD SENSORS AND ELECTRON DEVICES DIRECTORATE, ARMY RESEARCH LAB ADELPHI MD SENSORS AND ELECTRON DEVICES DIRECTORATE, 2010).

57. Liu, L. et al. High-Yield Chemical Vapor Deposition Growth of High-Quality Large-Area AB-Stacked Bilayer Graphene. ACS Nano6, 8241-8249 (2012).

58. Casiraghi, C., Pisana, S., Novoselov, K. S., Geim, A. K. \& Ferrari, A. C. Raman fingerprint of charged impurities in graphene. Appl. Phys. Lett.91, 233108 (2007).

59. Reina, A. et al. Large Area, Few-Layer Graphene Films on Arbitrary Substrates by Chemical Vapor Deposition. Nano Lett.9, 30-35 (2009).

60. Largeau, L.; Galopin, E.; Gogneau, N.; Travers, L.; Glas, F.; Harmand, J.-C. Cryst. Growth Des.2012, 12, 2724. 